\title{
Discontinuous Change in Membrane Activities of Plasmodium of Physarum polycephalum Caused by Temperature Variation: Effects on Chemoreception and Ameboid Motility
}

\author{
Tetsuo Ueda and Yonosuke Kobatake \\ Faculty of Pharmaceutical Sciences, Hokkaido University, Sapporo, Japan
}

\begin{abstract}
Variations in the surrounding temperature altered membrane activities at the critical temperature, $\mathrm{T}_{\mathrm{c}}=15^{\circ} \mathrm{C}$ in plasmodium of the true slime mold Physarum polycephalum. Changes in the membrane and zeta potentials, which were induced by chemical stimuli, decreased when the temperature decreased below $\mathrm{T}_{\mathrm{c}}$. The adsorption energy of a $-\mathrm{CH}_{2-}$ group on the membrane changed discretely by about $100 \mathrm{cal} / \mathrm{mol}$ at $T_{c}$. Decreasing the temperature sharply diminished the extension of psuedopodia by a factor of $1 / 10$ at $T_{c}$, while the motive force of protoplasmic streaming remained constant. This suggests that the formation of pseudopodia and the generation of motive force are different functions in ameboid motility. Results indicate that the surface membrane of the plasmodium undergoes a conformational change at $T_{c}$. The mechanisms of chemoreception and motility in plasmodium are discussed.
\end{abstract}

The temperature of the environment significantly influences living organisms. Bacteria, for example, undergo discrete changes in membrane structure which cause discontinuous variations in such activities as the growth rate, transport, respiration and chemotaxis $(5,6)$. Tetrahymena, a ciliate protozoan, contains different kinds of antigens depending on the growth temperature (12); the plasmodium of the true slime mold Physarum polycephalum may form sclerotia at a low $\left(5^{\circ} \mathrm{C}\right)$ temperature (13). These examples of variations due to surrounding temperature which produces physical and/or chemical changes in the structure and function of living organisms indicate that temperature changes can provide a powerful method for studying various biological activities.

Typical functions in the plasmodium of Physarum polycephalum, i.e., chemoreception, taxis and ameboid motility, are discussed. Chemoreception and taxis were examined by measuring changes in the membrane potential, electrophoretic mobility and the chemotactic motive force of protoplasmic streaming. Ameboid motility in the plasmodium was analyzed by comparing the extension of pseudopodia with the motive force of protoplasmic streaming. The plasmodium of Physarum polycephalum is shown to have two distinct stages in its membrane activities and the transition temperature is about $15^{\circ} \mathrm{C}$. These observations are discussed in relation to the mechanisms of chemoreception and motility in the plasmodium. 


\section{MATERIALS AND METHODS}

Measurements of changes in the membrane potential and motive force of protoplasmic streaming in the slime mold. Physarum polycephalum furnished by Prof. N. Kamiya of Osaka University was cultured by the method employed by Camp (4). The plasmodium was allowed to crawl overnight on $0.7 \%$ agar gel without feeding. The motive force of protoplasmic streaming, the chemotactic motive force and changes in membrane potential were measured by applying the double chamber method of Kamiya (14). Experimental details have been described in a previous paper (22). The basis of the method is the fact that the protoplasmic streaming can be controlled by applying a difference in hydrostatic pressure between two compartments. The averaged pressure difference, $\overline{\Delta \mathrm{P}}$, is taken as the motive force of chemotaxis of the plasmodium as illustrated in the upper trace in Fig. 4. where a negative sign of $\overline{\Delta \mathrm{P}}$ corresponds to a repellent. The potential difference between two compartments affords a measure of the membrane potential in so far as the potential change is concerned, since the plasmodial membrane in one compartment is always contiguous with a given standard solution $(10,22)$.

The hydrostatic pressure, $\Delta \mathrm{P}$, needed to counterbalance protoplasmic streaming was recorded by a pen-writing recorder through a pressure transducer (Statham Instruments, Inc., USA., Model SC 1001, Model UGP4). The double chamber was immersed in a water jacket adjusted to desired temperature. Experiments were performed three times for each concentration and temperature, then values were averaged for the data.

Measurements of the extension of pseudopodia in the plasmodium. The plasmodium was allowed to crawl on a filter paper before use. A small portion $(1 \mathrm{~cm} \times 1 \mathrm{~cm})$ on which the starved plasmodium was distributed densely was cut from the filter paper and was put on a $1 \%$ agar gel in a vessel $1 \mathrm{~cm}$ wide. After 30 to 60 min (according to the sample) the plasmodium began to extend pseudopodia on the agar gel. Time zero of the measurement was recorded at this stage. The inset in Fig. 1 illustrates how the plasmodium extended its

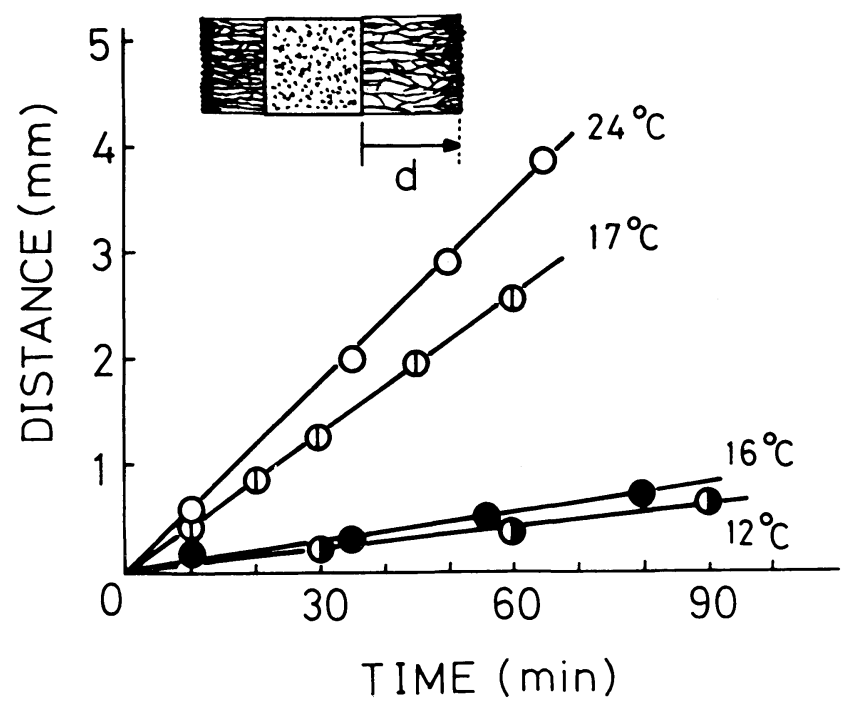

Fig. 1. Extension of pseudopodia in the plasmodium of Physarum polycephalum. Slopes in the plot give the rates of pseudopodium formation. 
pseudopodia. After the plasmodium had crawled on the agar gel, the distance of the spreading head portion was measured as a function of time. The plasmodium extended its pseudopodia linearly with time as shown in Fig. 1. The slopes give the rates of extension of the pseudopodia and were determined as a function of temperature. Average values are for three trials. When rates of pseudopodium extension at each side were different the faster rate was used as the rate of pseudopodium extension for this trial, because the slower side often retracted.

Measurements of the zeta potential for microplasmodia of Physarum polycephalum. Microplasmodia of the slime mold Physarum polycephalum, a gift from Prof. J. Ohta of Ochanomizu University, were cultured at $25^{\circ} \mathrm{C}$ with the method employed by Daniel and Rusch (6). Three- to four-day cultures were washed before use, then stored in a solution containing $10 \mathrm{mM}$ sucrose and $2 \mathrm{mM} \mathrm{NH}{ }_{4} \mathrm{Cl}$. Chemicals were added to this solution.

Electrophoretic mobilities, $u\left[\mathrm{~m}^{2} \mathrm{~V}^{-1} \mathrm{sec}^{-1}\right]$, were measured with a microelectrophoretic apparatus (Carl Zeiss, West Germany, Cytopherometer). Experimental details have been described previously (10). At each concentration and temperature, average values of u were determined for over 30 trials. The standard deviation was less than $1 \%$.

The zeta potential, $\zeta[\mathrm{v}]$, was determined from the observed electrophoretic mobility, $\mathrm{u}$, with the Helmholtz-Smoluchowski equation:

$$
\zeta=(4 \pi \eta / \varepsilon) \mathrm{u},
$$

where $\eta$ and $\varepsilon$ are the viscosity and dielectric constant of the medium, respectively.

\section{RESULTS}

Effect of temperature on chemoreception in the plasmodium of Physarum polycephalum. Fig. 2 shows the concentration dependence of changes in the membrane potential, $\Delta \varphi$, and in the zeta potential, $\zeta$, for $\mathrm{NaCl}$ and $\mathrm{CaCl}_{2}$ as typical examples at the different temperatures shown in the figure. Changes in the membrane and zeta potentials coincided at all the temperatures examined and changed linearly with $\log \mathrm{C}$ above the threshold concentration, $\mathrm{C}_{\mathrm{th}}$. Slopes in the $\Delta \varphi$ vs $\log \mathrm{C}$ plots for $\mathrm{NaCl}$ increased as the temperature rose. In contrast, those for $\mathrm{CaCl}_{2}$ changed discontinuously at a given point when the temperature rose successively. The critical temperature was about $15^{\circ} \mathrm{C}$.

Fig. 3 summarizes the temperature dependence of the magnitude of the response, $\mathrm{R}$, for various salts. Here, the magnitude of the response is defined by $\mathrm{R}=\partial(-\Delta \varphi) / \partial$ $\log \left(\mathrm{C} / \mathrm{C}_{\mathrm{th}}\right)$ or identically $\partial \zeta / \partial \log \left(\mathrm{C} / \mathrm{C}_{\mathrm{th}}\right)$ from the data shown in Fig. 2. Fig. 3a shows data for the monovalent cations; $\mathrm{NaCl}, \mathrm{KCl}, \mathrm{CsCl}$ and $\mathrm{LiCl}$. For these chemicals, the magnitude of the response did not change below $15^{\circ} \mathrm{C}$. It had a value of about $18 \mathrm{mV}$ for a ten-fold increase in concentration. Above $15^{\circ} \mathrm{C}$, changes in the membrane potential became larger as the temperature increased. This gave approximately straight lines for the different monovalent cations when $\mathrm{R}$ in the logarithmic scale was plotted as a function of temperature. Changes are largest for $\mathrm{NaCl}$ and are basically constant for $\mathrm{LiCl}$. In contrast, the values of $\mathrm{R}$ for the polyvalent cations $\mathrm{Ca}^{2+}, \mathrm{La}^{3+}$ and $\mathrm{Th}^{4+}$ had distinct values below and above the critical temperature (see Fig. $3 \mathrm{~b}$ ): $\mathrm{R}=4 \sim 5 \mathrm{mV}$ below $\mathrm{T}_{\mathrm{c}}$, and $\mathrm{R}=18 \mathrm{mV}$ above $\mathrm{T}_{\mathrm{c}} . \mathrm{SrCl}_{2}$, however, gave a constant value at all temperatures studied.

Results shown in Fig. 3 indicate that a critical temperature for chemoreception 

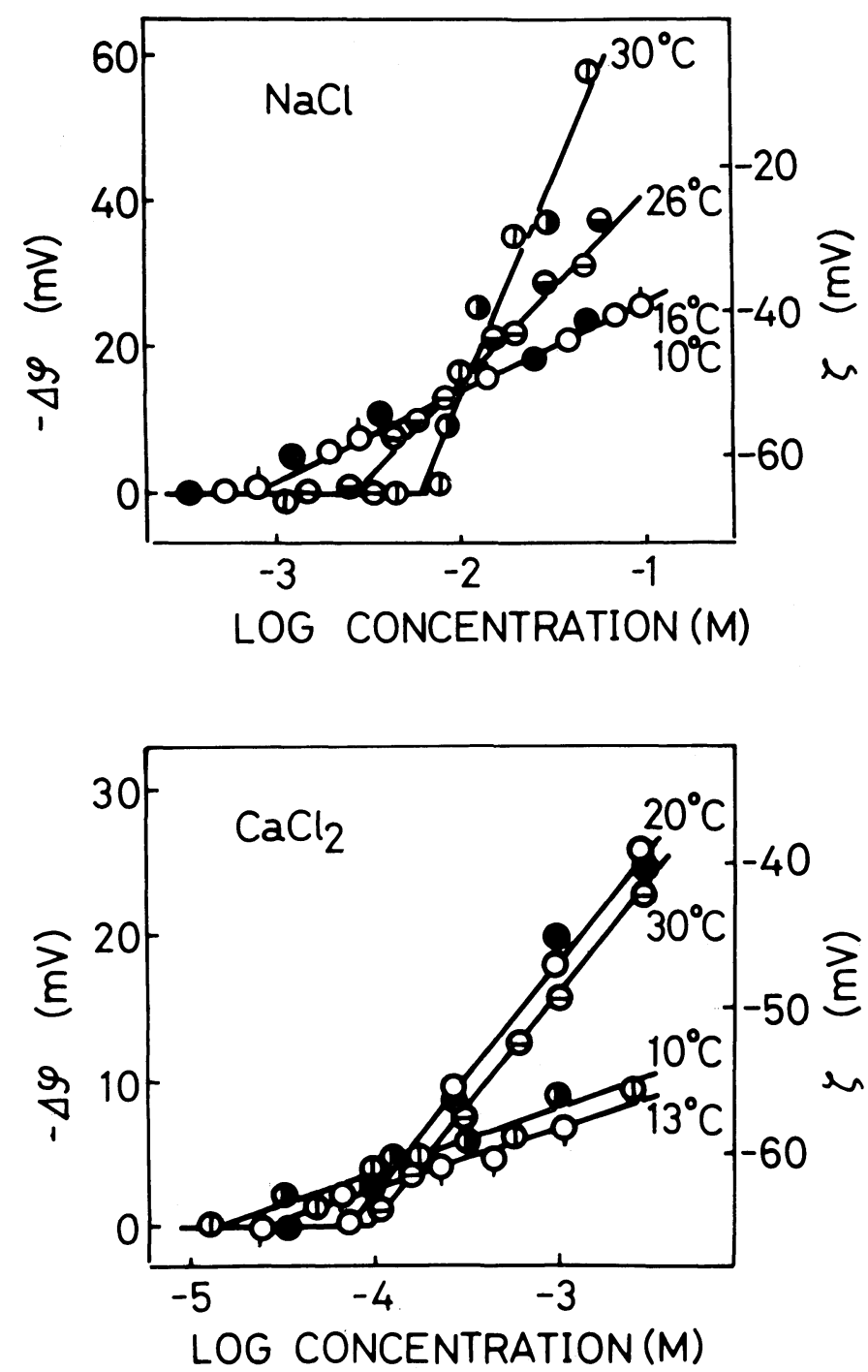

Fig. 2. Concentration dependence of changes in membrane and zeta potentials. (2a): Changes in membrane potential $(\circlearrowleft, \bigcirc, \ominus, \Phi)$ and in zeta potential $(\bullet, \ominus, \oplus)$ for $\mathrm{NaCl} .(2 \mathrm{~b})$ : Changes in membrane potential $(\odot, \oplus, \ominus, \bigcirc)$ and in zeta potential $(\bullet, \bullet)$ for $\mathrm{CaCl}_{2}$. Temperature are given in the figure.

exists in the plasmodium of Physarum polycephalum and that the receptive membrane has manifold characters when it reacts to chemical stimuli, e.g., the plasmodium discriminates $\mathrm{Ca}^{2+}$ and $\mathrm{Sr}^{2+}$ at the membrane potential level below $\mathrm{T}_{c}$, and a similar depolarization of the membrane potential is induced by $\mathrm{Ca}^{2+}$ and $\mathrm{Sr}^{2+}$ salts when $\mathrm{T}>\mathrm{T}_{\mathrm{c}}(10,22)$.

The fact that changes in the membrane potential agree with those in the zeta potential confirms the previous assertion that chemoreception in the plasmodium of Phy- 

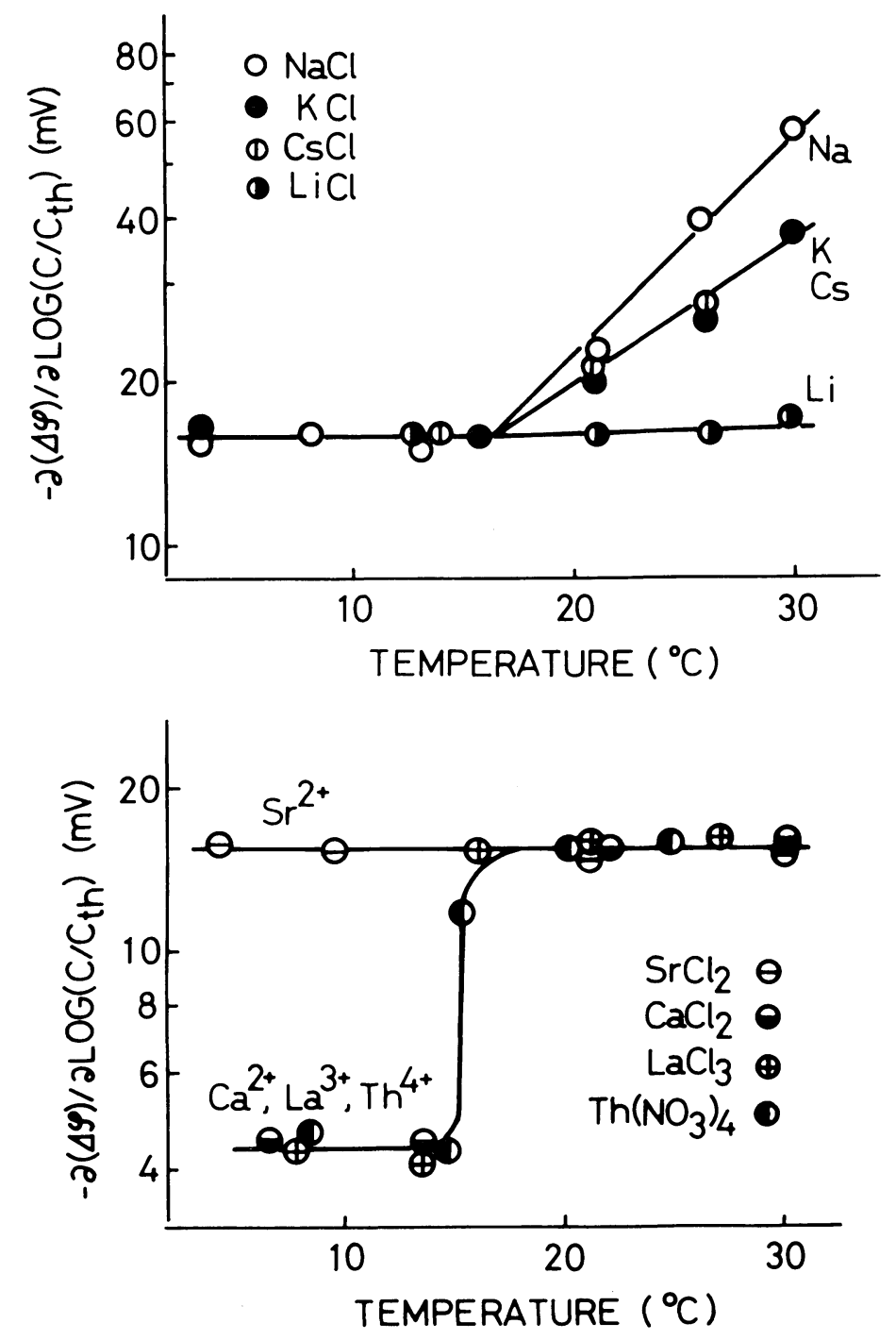

Fig. 3. Temperature dependence of the magnitude of the response $\mathrm{R}=\partial(-\Delta \psi) / \partial \log \left(\mathrm{C} / \mathbf{C}_{\mathrm{th}}\right)$.

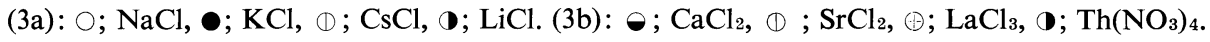

sarum polycephalum takes place at the membrane surface $(10,22)$. The temperature variations of the potentials (Fig. 3) is ascribed to changes in the surface charge density of the membrane, because the zeta potential is proportional to the surface charge density under the Debye-Hückel approximation (23). Therefore, the data shown in Fig. 2 mean that the surface charge density changes greatly above $T_{c}$, but is little influenced below $T_{c}$ in the reaction to chemical stimuli. The fact that temperature variation at a fixed concentration of a chemical induces a change in potential indicates that the density of the charges on the membrane surface varies with the surrounding temperature when $T$ is higher than $T_{c}{ }^{*}$ 
Temperature dependence of chemotaxis against $n$-alcohols. The upper trace of Fig. 4 shows the time course of the motive force of protoplasmic streaming in the plasmodium at $10^{\circ} \mathrm{C}$ when $100 \mathrm{mM}$ of 1-butanol was applied to one compartment of the double chamber at the time indicated by the arrow in the figure. The plasmodium moved away from the 1-butanol solution, and its motive force was counterbalanced by applying a difference in hydrostatic pressure, $\Delta \mathrm{P}$, as indicated in the figure. Removal of the chemical stimulus produced the original level of protoplasmic streaming.

The lower trace of Fig. 4 shows the concentration dependence of the chemotactic motive force, $\overline{\Delta \mathrm{P}}$ (the average of three trials), for $\mathrm{n}$-alcohols when $\overline{\Delta \mathrm{P}}$ was plotted
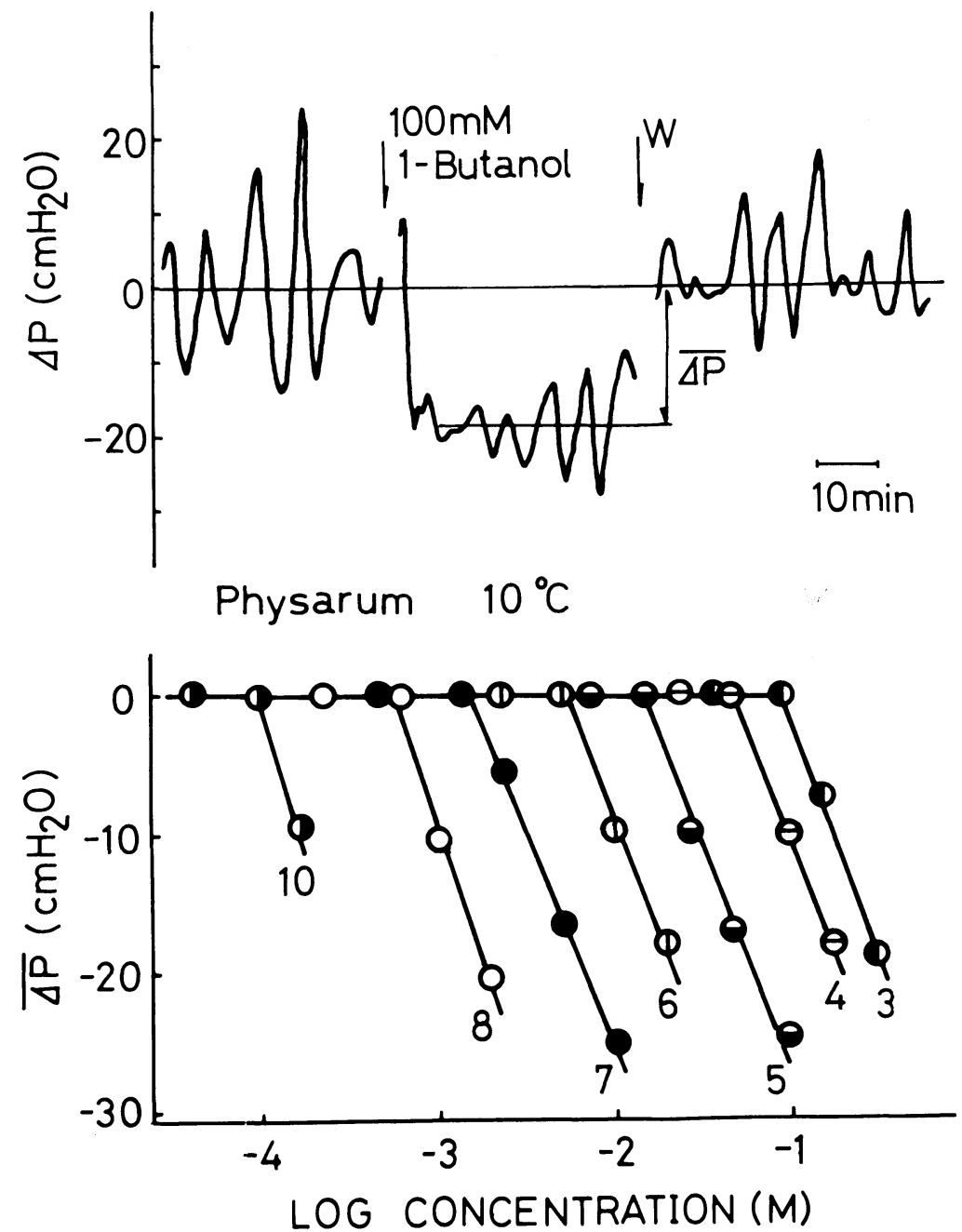

Fig. 4. Upper trace; time course of the motive force of protoplasmic streaming. $100 \mathrm{mM}$ of 1butanol was applied to one compartment at the time indicated by the arrow, then was washed out at the time indicated by the arrow with $\mathrm{W}$. The average deviation of $\Delta \mathrm{P}$ shows the chemotactic motive force, $\overline{\Delta \mathrm{P}}$. Lower trace; Concentration dependence of the chemotactic motive force, $\overline{\Delta \mathrm{P}}$, for $\mathrm{n}$-alcohols. The number of methylene groups in the compounds is shown in the figure. 
against the logarithm of concentration. The chemotactic motive force, $\overline{\Delta \mathrm{P}}$, increased linearly with $\log \mathrm{C}$ above the respective thresholds for $\mathrm{n}$-alcohols (21) This differs from the salts and sugar receptions where $\overline{\Delta \mathrm{P}}$ changed by $10 \mathrm{~cm}$ of $\mathrm{H}_{2} \mathrm{O}$ at the thresholds and remained constant with a further increase in concentration (22). Why this discrepancy in the behaviour of the slime mold against various chemical species exists is not clear. The threshold concentrations for $n$-alcohols were accurately determined by extrapolating the plots to $\overline{\Delta \mathrm{P}}=0$ in the $\overline{\Delta \mathrm{P}}$ vs $\log \mathrm{C}$ relation. We confirmed that no chemotactic movements took place at $\overline{\Delta \mathrm{P}}=0$.

Adsorption energy of a-CH2-group on the membrane of the plasmodium. The threshold concentration decreased systematically as the number of methylene groups in the compounds increased. Fig. 5 shows the plots of $\log C_{t h}$ against the number of methylene groups, $n$, in the $n$-alcohols below and above $T_{c}$. A linear relationship was obtained between $\log \mathrm{C}_{\mathrm{th}}$ and $\mathrm{n}$ as expressed by the following empirical relation:

$$
\log C_{t h}=-A \cdot n+B
$$

where $\mathrm{A}$ and $\mathrm{B}$ are constants.

The physical meaning of the parameter A and B in Eq. [2] can be clarified thus: Assume that a chemical component $\mathrm{i}$ is distributed between the aqueous and membrane phases when the plasmodium reacts to the chemical. The chemical potentials of $\mathrm{i}$ in the bulk and in the membrane phases are given by $\mu_{\mathrm{i}}{ }^{\mathrm{s}}$ and $\mu_{\mathrm{i}}{ }^{\mathrm{m}}$, respectively. At equilibrium, the chemical potential of $i$ in the two phases must be equal. Hence we have

$$
\mu_{\mathrm{i}}{ }^{\mathrm{os}}+\mathrm{RT} \ln \mathrm{C}_{\mathrm{i}}^{\mathrm{s}}=\mu_{\mathrm{i}}{ }^{\mathrm{om}}+\mathrm{RT} \ln \mathrm{C}_{\mathrm{i}}^{\mathrm{m}}
$$

where $\mu_{\mathrm{i}}{ }^{\text {os }}$ and $\mu_{\mathrm{i}}{ }^{\mathrm{om}}$ stand for the standard chemical potentials, and $\mathrm{C}_{\mathrm{i}}^{\mathrm{s}}$ and $\mathrm{C}_{\mathrm{i}}{ }^{\mathrm{m}}$ for the concentrations of $\mathrm{i}$ in the bulk solution and membrane phases, respectively. Rearranging Eq. [3] under the assumption that the difference in the standard chemical potential is proportional to the number of methylene groups, we obtain

$$
\ln \mathrm{C}_{\mathrm{i}}{ }^{\mathrm{s}}=-\left(\mu_{\mathrm{CH}_{2}}{ }^{\mathrm{os}}-\mu_{\mathrm{CH}_{2}}{ }^{o \mathrm{~m}}\right) \mathrm{n} / \mathrm{RT}+\ln \mathrm{C}_{\mathrm{i}}{ }^{\mathrm{m}}
$$

The assumption used to derive Eq. [4] has been confirmed in non-biological systems such as the oil/water partition equilibrium of n-alcohols (7).

A comparison of Eqs. [2] and [4] gives the physicochemical interpretation of parameters $\mathrm{A}$ and $\mathrm{B}$ :

$$
\begin{aligned}
& \mathrm{A}=\left(\mu_{\mathrm{CH}_{2}}{ }^{\mathrm{o}}-\mu_{\mathrm{CH}_{2}}{ }^{\mathrm{om}}\right) /(2.3 \mathrm{RT}) \\
& \mathrm{B}=\log \mathrm{C}_{\mathrm{i}}{ }^{\mathrm{m}}+\text { constant. }
\end{aligned}
$$

Eq. [5] states that parameter $\mathrm{A}$ is proportional to the difference in the standard chemical potential of a methylene group between the bulk solution and the membrane phases.

Since the hydrocarbon chain is a typical hydrophobic substance, the value of $A$ is a quantitative measure of the hydrophobicity of the membrane. Eq. [6] shows what concentration of a chemical, $i$, in the membrane is necessary to bring about a chemotactic response. Thus, parameter $B$ is closely correlated with the sensitivity of the membrane to chemical stimuli. Based on this analysis the hydrophobicity of biological 


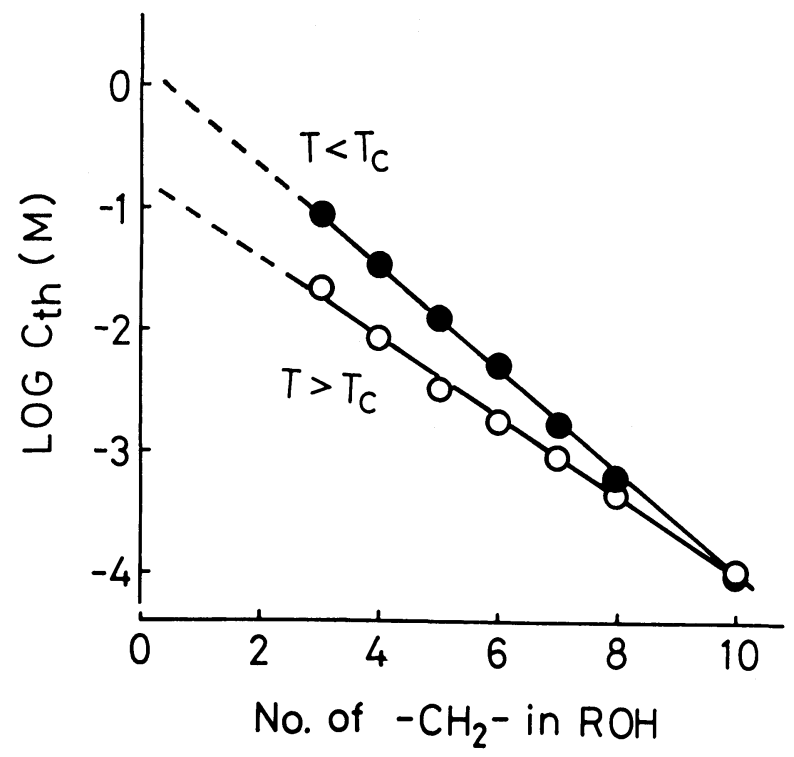

Fig. 5. Dependence of thresholds on the chain length for $n$-alcohols. close circles; $10^{\circ} \mathrm{C}$ (below the critical temperature $\mathrm{T}_{\mathrm{c}}=15^{\circ} \mathrm{C}$ ) open circles; 20 and $25^{\circ} \mathrm{C}$ (above the critical temperature $\mathrm{T}_{\mathrm{c}}$ )

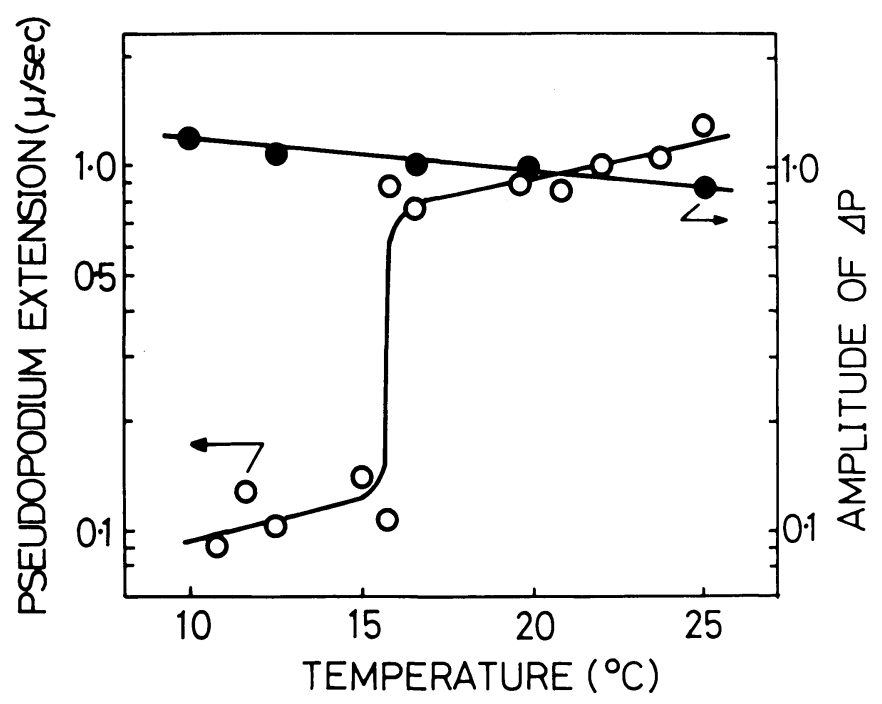

Fig. 6. Temperature dependence of the rate of pseudopodia extension and the amplitude of the motive force of protoplasmic streaming. open circles; pseudopodia extension, closed circles; amplitude of $\Delta \mathrm{P}$. 
membranes has been compared for various biological species as Tetrahymena, the internodal cell of Nitella, olfactory epithelia, etc. $(19,21)$.

The empirical relation described by Eq. [2] holds for all the temperature examined. Data obtained at $20^{\circ} \mathrm{C}$ and $25^{\circ} \mathrm{C}$ (above $\mathrm{T}_{\mathrm{c}}=15^{\circ} \mathrm{C}$ ) fall on a straight line in log $\mathrm{C}_{\text {th }}$ vs $n$ plot (see Fig. 5). Values of $\Delta \mu_{\mathrm{CH}_{2}}{ }^{\circ}\left(=\mu_{\mathrm{CH}_{2}}{ }^{\circ \mathrm{s}}-\mu_{\mathrm{CH}_{2}}{ }^{\circ \mathrm{m}}\right.$ ), as calculated by Eq. [5], are $580 \mathrm{cal} / \mathrm{mol}$ and $450 \mathrm{cal} / \mathrm{mol}$ below and above $T_{c}$, respectively. Due to the limitations of experimental accuracy, it is not clear whether the $\Delta \mu_{\mathrm{CH}_{2}}{ }^{\circ}$ obtained changes discontinuously at $\mathrm{T}_{\mathrm{c}}$. However, $\Delta \mu_{\mathrm{CH}_{2}}{ }^{\circ}$ alters by about $100 \mathrm{cal} / \mathrm{mol}$ below and above $\mathrm{T}_{\mathrm{c}}$. In other words, the surface membrane of the plasmodium becomes more hydrophobic below $T_{\mathrm{c}}$.

Temperature dependence of the extension of pseudopodia in Physarum polycephalum. Fig. 6 shows the temperature dependence of the extension of pseudopodia in Physarum polycephalum where the logarithm of the rate of pseudopodium extension is plotted against temperature. In the same figure, the amplitude of the motive force of protoplasmic streaming relative to that at $20^{\circ} \mathrm{C}$ is plotted by closed circles for comparison. This essentially confirms the results reported by Kamiya (15). The extension of the pseudopodium decreased by a factor of $1 / 10$ below $15^{\circ} \mathrm{C}$, but the amplitude of the motive force did not change appreciably at this critical temperature.

This shows that the extension of the pseudopodium and the generation of the motive force of protoplasmic streaming takes place at different and separate parts of the plasmodium and that each function may work independently. The implication of this statement for ameboid movement is discussed later. A comparison of Figs. 6 and 3 reveals that the critical temperature in chemoreception agrees with that for pseudopodia extension. This fact supports the view that pseudopodia extension is a membrane functions, as is chemoreception, generation of the membrane potential, etc.

\section{DISCUSSION}

The threshold and magnitude of the response in the chemoreception of Physarum polycephalum. Chemoreception in the plasmodium of Physarum polycephalum has duplicate characters. In previous papers, we have shown that the potential and tactic responses appear only above the thresholds and that these threshold points have a common feature with colloidal instabilities such as the Schulze-Hardy rule and the lyotropic number (21). Response to a chemical stimulus, A, was countered with a chemical, B, only when the concentration of B exceeded its threshold (18). This indicates that a certain discontinuity, or threshold, exists in the chemoreceptive processes, and that a continuous increase in the changes of the membrane potential occurs with an increase in stimulus concentration. The latter is discussed here.

Results summarized in Fig. 3 indicate that changes in the surface charge density of the membrane are primary process in the chemoreception of the plasmodium. The magnitude of the changes differed for different chemicals, which makes possible discrimination of chemical stimuli at the membrane potential level. The difference, however, does not correspond to positive and negative tactic movements $(20,21)$.

The thresholds, $\mathrm{C}_{\mathrm{th}}$, and the magnitude of the response, $\mathrm{R}=\partial(-\Delta \psi) / \partial \log \left(\mathrm{C} / \mathrm{C}_{\mathrm{th}}\right)$, exhibit different temperature dependences. The temperature dependence of $\mathrm{C}_{t h}$ was linear, with the same slope for all the chemicals examined in Arrhenius plots, i.e. log $\mathrm{C}_{\mathrm{th}}$ vs $1 / \mathrm{T}(21)$. In contrast, $\mathrm{R}$ shows a transition point with temperature and has 
different values for different chemicals. Thus, the threshold and the potential change in chemoreception may be regarded as separate membrane functions.

Generation of the motive force of protoplasmic streaming and the pseudopodia extension in the plasmodium of Physarum polycephalum. We previously reported that chemotaxis in Physarum polycephalum can be quantitatively measured as the average deviation of the motive force of protoplasmic streaming, $\overline{\Delta \mathrm{P}}$, and that the positive and negative signs of $\overline{\Delta \mathrm{P}}$ are correlated with the relaxation and contraction of the plasmodial strands, respectively, when a chemical stimulus is applied locally (20). These facts indicate the indispensable role of motive force in the locomotion of the plasmodium and in ameboid motility, in general.

Organisms with ameboid motility, including Physarum polycephalum, expand their surface membranes to form pseudopodia for locomotion. Thus, many studies of ameboid movement have paid special attention to the formation of pseudopodia $(1,3)$. The relationship between pseudopodia formation and the generation of the motive force of protoplasmic streaming is, however, slightly understood at present. Results shown in Fig. 6 cast some light on this relationship. Pseudopodia formation and the generation of the motive force may be regarded as separate functions. DuPraw reported essentially similar results for the ameboid movement of honey bee embryoic cells observed by cinematography (8).

Pseudopodia formation and the generation of the motive force of protoplasmic streaming seem to work cooperatively or additively in favor of locomotion. In the galvanotaxis of Physarum polycephalum, Anderson reported that the extension of pseudopodia on the anodal side is inhibited while that on the cathodal side is not (2). The plasmodium, as a result, migrates to the cathodal side. We have observed that a $\overline{\Delta \mathrm{P}}$ of about $10 \mathrm{~cm} \mathrm{H}_{2} \mathrm{O}$ is generated by passing the current above the threshold strength, which tends to move the plasma sol to the cathodal side. The same holds for thermotaxis and phototaxis. A plasmodium in a temperature gradient moves to the warmer side by expanding its pseudopodia $(9,17)$. This expansion is accelerated by a $\overline{\Delta \mathrm{P}}$ of more than $10 \mathrm{~cm} \mathrm{H} \mathrm{H}_{2} \mathrm{O}$ (present study). The plasmodium is known to move away from a white light by retracting its pseudopodia. An extensive study on phototaxis was performed by Hato et al. with monochromatic light (11). Blue light, for example, induces negative taxis in the plasmodium, contraction of the plasmodial strands and the generation of $\overline{\Delta \mathrm{P}}$ which tends to produce movement away from the light portion. Further study is necessary to determine the mechanism by which pseudopodia formation and the generation of $\overline{\Delta \mathrm{P}}$ work cooperatively.

Acknowledgements. We are deeply indebted to Mr. T. Nakazawa for performing part of the experiments reported here. Thanks are also due to Prof. emeritus N. Kamiya of Osaka University and Prof. J. Ohta of Ochanomizu University for furnishing the organisms.

\section{REFERENCES}

1. Allen, R.D. Biophysical aspects of pseudopodium formation and retraction. In The Biology of Amoeba, ed. JeOn, K.W., Academic Press, New York and London: pp. 201-247, 1973

2. Anderson, J.D. Galvanotaxis of slime mold. J. Gen. Physiol. 35, 1-16, 1951

3. Bovee, E.C. and T.L. JAHN. Locomotion and behaviour. In The Biology of Amoeba, ed. JEON, K.W., Academic Press, New York and London: pp. 250-283, 1973

4. CAMP, W.G. A method of cultivating myxomycete plasmodia. Bull. Torry Bot. Club. 63, 205210,1936 
5. Cronan, J.E. and E.P. Gelmann. Physical properties of membrane lipids: biological relevance and regulation. Bacteriol. Rev. 39, 232-256, 1975

6. DANIEL, J.W. and H.P. RuSCH. The pure culture of Physarum polycephalum on a partially defined soluble medium. J. Gen. Microbiol. 25, 47-59, 1961

7. Davies, J.T. and E.K. RedeAL. Interfacial Phenomena. Academic Press, New York and London, 1961

8. DuPraw, E.J. The organization of honey bee embryonic cells. I. microtubles and amoeboid activity. Devel. Biol. 12, 53-71, 1965

9. Durham, A.C.H. and E.B. Ridgway. Control of chemotaxis in Physarum polycephalum. J. Cell Biol. 69, 218-223, 1976

10. Hato, M., T. Ueda, K. Kurihara and Y. Kobatake. Changes in zeta potential and membrane potential of slime mold Physarum polycephalum in response to chemical stimuli. Biochim. Biophys Acta 426, 73-80, 1976

11. Hato, M., T. Ueda, K. Kurihara and Y. Kobatake. Phototaxis in Physarum polycephalum. Cell Struct. \& Funct. 1, 269-278, 1976

12. Hill, D.L. The Biochemistry and Physiology of Tetrahymena. Academic Press, New York and London. 1972

13. Jump, J.A. Studies on sclerotization in Physarum polycephalum. Am. J. Bot. 41, 561-567, 1954

14. KamiYA, N. Physical aspects of protoplasmic streaming. In The Structure of Protoplasms. ed. SeIfriz, W., pp. 199-244, Iowa: Iowa State University Press, 1942

15. KamiYa, N. Protoplasmic streaming. Protoplasmatologia 8, 3a, 1-199, 1959

16. Lofgren, K.W. and C.F. Fox. Attractant-directed motility in Escherichia coli: requirement for a fluid lipid phase. J. Bacteriol. 118, 1181-1182, 1974

17. Stahl, E. Zur Biologie der Myxomyceten. Bot. Zeit. 42, 145-156, 1884

18. Terayama, K., T. Ueda, K. Kurihara and Y. Kobatake. Effect of sugars on salt reception in true slime mold Physarum polycephalum, Physicochemical interpretation of interaction between salt and sugar receptions. J. Membrane Biol. 34, 369-381, 1977

19. Ueda, T. and Y. Kobatake. Hydrophobicity of biosurfaces as shown by chemoreceptive thresholds in Tetrahymena, Physarum and Nitella. J. Membrane Biol. 34, 351-368, 1977

20. Ueda, T., M. Muratsugu, K. Kurihara and Y. Kobatake. Chemotaxis in Physarum polycephalum: Effects of chemicals on isometric tension of the plasmodial strand in relation to chemotactic movement. Exp. Cell Res. 100, 337-344, 1976

21. UedA, T. and Y. KobataKe. Changes in membrane potential, zeta potential and chemotaxis of Physarum polycephalum in response to n-alcohols, n-aldehydes and n-fatty acids. Cytobiologie 16, 16-26, 1977

22. Ueda, T., K. Terayama, K. Kurihara and Y. Kobatake. Threshold phenomena in chemoreception and taxis in slime mold Physarum polycephalum. J. Gen. Physiol. 65, 223-234, 1975

23. Verway, E.J. and J. Th G. Overbeek. Theory of Stability of Lyophobic Colloids. Amsterdam: Elsevier, 1948 